\title{
A Computable Formula for the Average Bit Error Probability as a Function of Window Size for the IEEE 802.15.3a UWB Channel Model
}

\author{
John A. Gubner, Member, IEEE, and Kei Hao
}

\begin{abstract}
Binary signaling over the IEEE 802.15.3a ultra-wideband channel model is considered. For received waveforms observed during a finite time window, a formula is derived for the average bit error probability (BEP). The formula can be rapidly evaluated using Gaussian quadratures, and is used to study average BEP curves as a function of window size, which is then related to the percentage of energy captured. These techniques should be useful to system designers in assessing the performance impact of various signaling rates, guard times, etc.
\end{abstract}

Index Terms-Cluster process, lognormal distribution, point process, Saleh-Valenzuela model, shot noise, ultra-wideband (UWB).

\section{INTRODUCTION}

$\mathbf{T}$ HE IEEE 802.15.3a standards body [5] has developed a modification of the Saleh-Valenzuela multipath channel model [16] as the accepted model for ultra-wideband (UWB) channel investigations [2], [12]. According to this mathematical model, multipath copies of a transmitted signal arrive at the receiver at times $T_{k}$ according to a point process defined in terms of various homogeneous (constant intensity) Poisson processes. Consequently, there is an infinite sequence of arrival times $T_{0}=0<T_{1}<T_{2}<\cdots$ with $T_{k} \rightarrow \infty$ as $k \rightarrow \infty$. Corresponding to each arrival time $T_{k}$, there is a channel gain $G_{k}$. Since $T_{k} \rightarrow \infty$, to capture all the signal energy, we theoretically need an observation window of infinite length. However, we are fortunate that $G_{k}$ depends on $T_{k}$ in such a way that for large values of $T_{k}, G_{k}$ is negligibly small (with high probability). Thus, as a practical matter, it is only necessary to observe the gains that arrive in a finite time window, say, $[0, T]$.

We are interested in what can be said about how large $T$ should be to achieve certain goals. For example, simple formulas for the expected number of paths in a time window have been given in [6]. Hence, it would be easy to choose $T$ so that the expected number of paths exceeds some threshold. As another example, [6] gives closed-form expressions for the expected sum of squares of the gains that arrive in a given time window, including the limiting case of the semi-infinite window $[0, \infty)$. Hence, it is easy to determine the value of $T$ so that the expected energy in $[0, T]$ is a required fraction of the expected energy in $[0, \infty)$.

Manuscript received July 27, 2005; revised January 9, 2006. This paper was presented in part at the 2005 IEEE International Conference on Ultra-Wideband.

The authors are with the Department of Electrical and Computer Engineering, University of Wisconsin-Madison, Madison, WI 53706-1691 USA (e-mail: gubner@engr.wisc.edu; khao@wisc.edu).

Digital Object Identifier 10.1109/TMTT.2006.871985
In this paper, we derive a computable formula for the average bit error probability (BEP) for binary signaling over the IEEE 802.15.3a channel model when the received waveform is observed only over a finite window $[0, T]$. We show that as $T \rightarrow \infty$, our formula reduces to the one derived for $[0, \infty)$ in [7]. We then present BEP curves for increasing values of $T$ and show how they converge to the limiting BEP curve for $[0, \infty)$. Our techniques should prove useful to system designers in assessing the performance impact of various signaling rates, guard times, etc.

The specifications of the Saleh-Valenzuela [16] model and its IEEE 802.15.3a modification [2], [5], [12] are presented in a way that makes them easy to simulate, but challenging to analyze theoretically. Hence, most studies are done using Monte Carlo methods, e.g., [8], [9], [11], [14], [15], [18], and [19]. In contrast, our results here require only a modest amount of numerical integration; no simulation is needed.

\section{A. Outline of this Paper}

We begin by giving a precise formulation of our problem in Section II. After presenting a general formula for the average BEP, we apply Craig's formula to obtain an integral that is easily computable using Chebyshev-Gauss quadrature, provided that we can also compute the moment generating function of the sum of the squares of the gains that arrive in $[0, T]$.

To facilitate the determination of the required moment generating function, in Section III, we show that the sum of the squares of the gains in $[0, T]$ can be viewed as a shot-noise random variable driven by a two-dimensional point process that is implicitly defined by the IEEE 802.15.3a model. This observation is then exploited in Section IV to derive a formula for the moment generating function that can be computed easily using Hermite-Gauss and Legendre-Gauss quadratures.

Numerical examples are given in Section $\mathrm{V}$, and a conclusion is presented in Section VI.

\section{Problem Formulation}

Frequency-selective fading channels are well modeled by time-varying impulse responses of the form [13]

$$
h(t, \tau)=\sum_{k} G_{k}(t) \delta\left(\tau-T_{k}(t)\right)
$$

where $t$ and $\tau$ are the observation time and the application time of the impulse, respectively. Here, $G_{k}(t)$ are the time-varying gains and $T_{k}(t)$ are the time-varying path arrival times. Since we 
focus on indoor environments whose structure changes slowly in comparison with the signaling rate, we use the corresponding time-invariant model

$$
h(\tau)=\sum_{k} G_{k} \delta\left(\tau-T_{k}\right)
$$

The response of such a channel to a waveform $\xi(t)$ is

$$
\hat{\xi}(t)=\sum_{k} G_{k} \xi\left(t-T_{k}\right)
$$

where $T_{k} \geq 0$. If the receiver only observes the waveform over the window $[0, T]$, then the received signal is given by

$$
\tilde{\xi}(t)=\hat{\xi}(t) I_{[0, T]}(t)
$$

where $I_{[0, T]}(\cdot)$ is the indicator function of the set $[0, T]$, i.e., $I_{[0, T]}(t):=1$ if $t \in[0, T]$ and $I_{[0, T]}(t):=0$ if $t \notin[0, T]$.

If we also account for additive white Gaussian noise of power spectral density $\sigma_{n}^{2}$ at the receiver, and then any signal detection is based on the waveform

$$
\rho(t)=\tilde{\xi}(t)+n(t)
$$

In a binary signaling scheme, there will be two transmitted signals $\xi_{i}(t), i=0,1$, and two received signals $\tilde{\xi}_{i}(t)$. Since $\xi_{i}(t)$ are known, conditioned on the gains $G_{k}$ and arrival times $T_{k}$, the received signals $\tilde{\xi}_{i}(t)$ are also known. Hence, the conditional probability of error is 1

$$
Q\left(\sqrt{d^{2} /\left(4 \sigma_{n}^{2}\right)}\right)
$$

where $Q(x):=\int_{x}^{\infty} e^{-t^{2} / 2} / \sqrt{2 \pi} d t$ is the standard normal complementary cumulative distribution function, and $d$ is the distance between the received waveforms $\tilde{\xi}_{1}$ and $\tilde{\xi}_{0}$. If we put

$$
\tilde{\xi}_{\Delta}(t):=\tilde{\xi}_{1}(t)-\tilde{\xi}_{0}(t)
$$

then

$$
d^{2}=\int\left|\tilde{\xi}_{\Delta}(t)\right|^{2} d t
$$

Now put

$$
\xi_{\Delta}(t):=\xi_{1}(t)-\xi_{0}(t) .
$$

${ }^{1}$ In the IEEE 802.15.3a model, the gains $G_{k}$ are real, as is the additive noise.
We assume that $\xi_{i}$ are short-duration pulses on $\left[0, t_{p}\right]$, where $t_{p} \ll T$. Hence, $\xi_{\Delta}(t)=0$ for $t \notin\left[0, t_{p}\right]$ and, thus,

$$
\begin{aligned}
d^{2}= & \sum_{k} \sum_{l} G_{k} G_{l} \int_{0}^{T} \xi_{\Delta}\left(t-T_{k}\right) \xi_{\Delta}\left(t-T_{l}\right) d t \\
= & \sum_{k} \sum_{l} G_{k} G_{l} I_{\left[0, t_{p}\right]}\left(\left|T_{l}-T_{k}\right|\right) \\
& \cdot \int_{0}^{T} \xi_{\Delta}\left(t-T_{k}\right) \xi_{\Delta}\left(t-T_{l}\right) d t .
\end{aligned}
$$

If $t_{p}$ is small enough that for any pair $k \neq l$ the probability of $\left|T_{l}-T_{k}\right| \leq t_{p}$ is negligible, then

$$
\begin{aligned}
d^{2} & \approx \sum_{k} G_{k}^{2} \int_{0}^{T} \xi_{\Delta}^{2}\left(t-T_{k}\right) d t \\
& =\underbrace{\sum_{k} G_{k}^{2} I_{[0, T]}\left(T_{k}\right)}_{=: \Phi} \int_{T_{k}}^{\min \left(T_{k}+t_{p}, T\right)} \xi_{\Delta}^{2}\left(t-T_{k}\right) d t .
\end{aligned}
$$

Note that we have suppressed the dependence of $\Phi$ on $T$. If we assume the probability that any $T_{k}$ lies in $\left[T-t_{p}, T\right]$ is negligible, then the integral in (3) is approximately equal to

$$
\int_{T_{k}}^{T_{k}+t_{p}} \xi_{\Delta}^{2}\left(t-T_{k}\right) d t=\int_{0}^{t_{p}}\left|\xi_{\Delta}(t)\right|^{2} d t=: \mathcal{E}
$$

which is the energy of the waveform $\xi_{\Delta}(t)$. We thus have

$$
d^{2} \approx \mathcal{E} \Phi
$$

If we substitute this approximation in (1), we see that the average BEP is approximately

$$
\mathrm{E}\left[Q\left(\sqrt{\frac{\mathcal{E} \Phi}{4 \sigma_{n}^{2}}}\right)\right]
$$

At this point, as suggested in [17, p. 1864], we exploit Craig's formula [3, p. 572, eq. (9)]

$$
Q(x)=\frac{1}{\pi} \int_{0}^{\frac{\pi}{2}} \exp \left(\frac{-x^{2}}{2 \sin ^{2} t}\right) d t .
$$

By making the change of variable $y=\sin t$,

$$
Q(x)=\frac{1}{\pi} \int_{0}^{1} \frac{\exp \left(-x^{2} / 2 y^{2}\right)}{\sqrt{1-y^{2}}} d y
$$


Since the integrand is an even function, we can write

$$
Q(x)=\frac{1}{2 \pi} \int_{-1}^{1} \frac{\exp \left(-x^{2} / 2 y^{2}\right)}{\sqrt{1-y^{2}}} d y .
$$

Using this in (4) and interchanging the expectation and integral, we obtain

$$
P_{T}(\eta):=\frac{1}{2 \pi} \int_{-1}^{1} \frac{\mathrm{E}\left[e^{-\eta \Phi / y^{2}}\right]}{\sqrt{1-y^{2}}} d y=\frac{1}{2 \pi} \int_{-1}^{1} \frac{M\left(-\eta / y^{2}\right)}{\sqrt{1-y^{2}}} d y
$$

where

$$
\eta:=\frac{\mathcal{E}}{8 \sigma_{n}^{2}}
$$

is the signal-to-noise ratio and

$$
M(\theta):=\mathrm{E}\left[e^{\theta \Phi}\right], \quad \theta \leq 0
$$

is the moment generating function of the random variable $\Phi$, which is discussed in Section III. The advantage of writing the average BEP in the form of (5) is that it is in the form for the Chebyshev-Gauss quadrature [1, p. 889]. Thus,

$$
P_{T}(\eta) \approx \frac{1}{2 K} \sum_{k=1}^{K} M\left(-\eta / y_{k}^{2}\right)
$$

where

$$
y_{k}=\cos \left[\frac{(2 k-1) \pi}{2 K}\right]
$$

and $K$ is the number of weights to evaluate the quadrature. If $K$ is an odd number, $y_{k}$ is zero for $k=(K+1) / 2$ (the middle point); thus the argument of the moment generating function (MGF) goes to $-\infty$. Now use the fact that $\lim _{\theta \rightarrow-\infty} M(\theta)=$ 0 for strictly positive random variables. Furthermore, the remaining points form pairs of numbers with opposite sign, and they are symmetric with respect to the middle point. Therefore, only the first $(K-1) / 2$ points are needed for the evaluation. Hence,

$$
P_{T}(\eta) \approx \frac{1}{K} \sum_{k=1}^{(K-1) / 2} M\left(-\eta / y_{k}^{2}\right)
$$

\section{III. $\Phi$ AND THE IEEE 802.15.3A UWB CHANNEL MODEL}

We begin with the observation that if $\varphi(s, g)=g^{2} I_{[0, T]}(s)$, then $\Phi$ in (3) is a special case of the counting integral

$$
\int_{0}^{\infty} \int_{-\infty}^{\infty} \varphi(s, g) N(d s \times d g):=\sum_{k} \varphi\left(T_{k}, G_{k}\right)
$$

where $N(\cdot)$ is the counting measure on $[0, \infty) \times(-\infty, \infty)$ that puts a unit mass at each point $\left(T_{k}, G_{k}\right)$, where the arrival times $T_{k}$ and path gains $G_{k}$ are specified by the IEEE 802.15.3a model. A random variable of the form in (7) is called a shotnoise random variable.

The IEEE 802.15.3a model [2] is a modification of the Saleh-Valenzuela model [16]. In these models, paths arrive in clusters, as described in the rest of this section.

\section{A. Distribution of the Initial Paths of the Clusters}

The initial path of the initial cluster arrives at time zero with a gain $G_{0}$ having a probability density that we denote by $f_{0,0}(\cdot)$.

Next, independent of $G_{0}$, the arrival times of the initial paths of the remaining clusters are modeled as a homogeneous Poisson process of constant intensity $C$, which is called the cluster arrival rate. If the initial path of such a cluster arrives at time $\tau$, its gain has a density that we denote by $f_{\tau, \tau}(\cdot)$. The gains of different initial paths are independent and can be considered marks of the Poisson arrival times. Such a marked Poisson process is equivalent to a two-dimensional Poisson process with intensity function [10, Sec. 5.2]

$$
\lambda_{1}(\tau, \gamma):=C f_{\tau, \tau}(\gamma), \quad \tau \geq 0, \gamma \in \mathbb{R}
$$

\section{B. Distribution of Noninitial Paths of the Clusters}

Conditional on the arrival times of the initial paths of the clusters, the arrival times of the noninitial paths of different clusters, including the cluster that starts at time zero, are modeled as independent homogeneous Poisson processes. Each of these Poisson processes has the same constant intensity $R$, which is called the ray arrival rate. If one of these paths arrives at time $s$ and is part of a cluster that started at time $\tau$, the arriving path gain has a density that we denote by $f_{\tau, s}(\cdot)$. These gains are independent and can be considered marks of the Poisson arrival times of the noninitial paths in the cluster. As mentioned above, such a marked Poisson process is equivalent to a two-dimensional Poisson process. Here, the intensity function is ${ }^{2}$

$$
\lambda_{r}(s, g \mid \tau, \gamma):=R f_{\tau, s}(g) I_{[\tau, \infty)}(s) .
$$

Note that $\lambda_{r}(s, g \mid \tau, \gamma)$ depends on $\tau$, but not $\gamma$.

\section{Assumptions About the Densities of the Gains}

Following Saleh and Valenzuela [16, eq. (26)] and Batra et al. [2, p. 2126], we assume $f_{\tau, s}(\cdot)$ has second moment

$$
\Omega_{0} e^{-\tau / \tau_{0}} e^{-(s-\tau) / s_{0}}
$$

where $\tau_{0}$ and $s_{0}$ are power-delay time constants and $\Omega_{0}$ is a scale factor. In [16], $f_{\tau, s}(\cdot)$ is taken to be a Rayleigh density. For the IEEE 802.15.3a model in [2], a $\{ \pm 1\}$-valued-Bernoulli(1/2)

\footnotetext{
${ }^{2}$ Since the intensity in (9) is zero for $s<\tau$, the Poisson process starts at time $\tau$. This is in contrast to [2] and [16]. Their ray processes were defined by taking Poisson processes starting at time zero and then translating them by the arrival time of the initial path in the cluster. The two constructions are equivalent provided we adjust the definition of $f_{\tau, s}(\cdot)$. This is done in (10) where we use $s-\tau ;[2]$ and [16] would use only $s$.
} 
mixture of lognormal densities is used. This implies that if $G$ has density $f_{\tau, s}(\cdot)$, then $10 \log _{10} G^{2}$ is normal with mean

$$
\mu_{\tau, s}:=\frac{10}{\ln 10}\left[\ln \Omega_{0}-\frac{\tau}{\tau_{0}}-\frac{(s-\tau)}{s_{0}}-\left(\frac{\ln 10}{10}\right)^{2} \frac{\sigma^{2}}{2}\right]
$$

and variance $\sigma^{2}$. Hence,

$$
\mathrm{E}_{\tau, s}\left[e^{\theta G^{2}}\right]=\int_{-\infty}^{\infty} \exp \left[10^{\left(x+\mu_{\tau, s}\right) / 10} \theta\right] \frac{e^{-(x / \sigma)^{2} / 2}}{\sqrt{2 \pi} \sigma} d x
$$

\section{Moment Generating Function of $\Phi$}

It is shown in [6] that under the IEEE 802.15.3a model described above, a counting integral of the form (7) can be written as the sum of the three statistically independent terms

$$
\Phi=\varphi\left(0, G_{0}\right)+\Phi_{r 0}+\Phi_{\otimes}
$$

where $\Phi_{r 0}$ and $\Phi_{\otimes}$ are defined in the Appendix. Hence,

$$
M(\theta):=\mathrm{E}\left[e^{\theta \Phi}\right]=\mathrm{E}\left[e^{\theta \varphi\left(0, G_{0}\right)}\right] \mathrm{E}\left[e^{\theta \Phi_{r 0}}\right] \mathrm{E}\left[e^{\theta \Phi_{\otimes}}\right] .
$$

It is shown in the Appendix that

$$
M(\theta)=M_{\sigma}\left(K_{0} \theta\right) e^{-R \psi_{0}\left(K_{0} \theta\right)} e^{-C J\left(K_{0} \theta\right)}
$$

where

$$
\begin{aligned}
K_{0} & :=\Omega_{0} \exp \left[-\left(\frac{\ln 10}{10}\right)^{2} \frac{\sigma^{2}}{2}\right] \\
M_{\sigma}(\theta) & :=\frac{1}{\sqrt{\pi}} \int_{-\infty}^{\infty} \exp \left[10^{\sqrt{2} \sigma x / 10} \theta\right] e^{-x^{2}} d x \\
\psi_{\tau}(\theta) & =(T-\tau) \int_{0}^{1} 1-M_{\sigma}\left(\theta e^{-x(T-\tau) / s_{0}}\right) d x
\end{aligned}
$$

and

$$
\begin{aligned}
& J(\theta)=T \int_{0}^{1} 1-M_{\sigma}\left(\theta e^{-x T / \tau_{0}}\right) \\
& \cdot \exp \left(-R \psi_{x T}\left(\theta e^{-x T / \tau_{0}}\right)\right) d x .
\end{aligned}
$$

We then use the fact that (16) is in the form for the Hermite-Gauss quadrature [1, p. 890], while (17) and (18) are in the form for the Legendre-Gauss quadrature on $[0,1][1, p$. 887, form. 25.4.30]. Hence, it is relatively straightforward to compute $M(\theta)$ and then the average BEP in (6).

\section{A. Results for an Infinite Observation Window}

To evaluate the average BEP for an infinite observation window $(T=\infty)$, we need to compute $\tilde{M}(\theta):=$ $\lim _{T \rightarrow \infty} M(\theta)$.

Let $\tilde{\psi}(\theta):=\lim _{T \rightarrow \infty} \psi_{\tau}(\theta)$. Rather than use (17), we use the alternate form (22) in the Appendix. Taking the limit in (22) and then making the change of variable $t=s-\tau$, it is easy to see that the limit does not depend on $\tau$. However, to facilitate the numerical evaluation of $\tilde{\psi}(\theta)$, it is more convenient to make the change of variable $x=\exp \left[-(s-\tau) / s_{0}\right]$. Thus,

$$
\tilde{\psi}(\theta)=s_{0} \int_{0}^{1} \frac{1-M_{\sigma}(\theta x)}{x} d x
$$

which is suited to the Legendre-Gauss quadrature.

Let $\tilde{J}(\theta):=\lim _{T \rightarrow \infty} J(\theta)$. Rather than use (18), we use the alternate form in (25). In taking the limit in (25), we must keep in mind that the function $\psi_{\tau}$ depends on $T$. Thus,

$$
\begin{aligned}
\tilde{J}(\theta) & =\int_{0}^{\infty} 1-M_{\sigma}\left(\theta e^{-\tau / \tau_{0}}\right) \exp \left[-R \tilde{\psi}\left(\theta e^{-\tau / \tau_{0}}\right)\right] d \tau \\
& =\tau_{0} \int_{0}^{1} \frac{1-M_{\sigma}(\theta x) e^{-R \tilde{\psi}(\theta x)}}{x} d x
\end{aligned}
$$

where the last step uses the change of variable $x=e^{-\tau / \tau_{0}}$. Note that the result is suited to the Legendre-Gauss quadrature. We can now compute the limit of $M(\theta)$ in (14) as $T \rightarrow \infty$. We find that

$$
\tilde{M}(\theta):=\lim _{T \rightarrow \infty} M(\theta)=M_{\sigma}\left(K_{0} \theta\right) e^{-R \tilde{\psi}\left(K_{0} \theta\right)} e^{-C \tilde{J}\left(K_{0} \theta\right)}
$$

and the average BEP can be computed by replacing $M(\cdot)$ with $\tilde{M}(\cdot)$ in (6). Note that (21) is the moment generating function derived in [7].

\section{NUMERICAL EXAMPLES}

We have derived formulas for the numerical computation of the average BEP $P_{T}(\eta)$. We now apply these formulas to study $P_{T}(\eta)$ as a function of the receiver's observation window size $T$.

The channel parameters we consider are shown in Table I. These parameters describe four UWB channels studied in [5]. Fig. 1 shows $P_{T}(\eta)$ for increasing values of $T$, with the solid line being $T=\infty$. For CM1, the other values of $T$ are 12, 16, and $26 \mathrm{~ns}$. For CM2, the other values of $T$ are 20, 25, and $37 \mathrm{~ns}$. For CM3, the other values of $T$ are 33, 43, and 65 ns. For CM4, the other values of $T$ are 59, 76, and $115 \mathrm{~ns}$.

Recall that $\Phi$ in (3) is the sum of the squares of the channel gains that arrive in $[0, T]$. The window sizes $T$ used in Fig. 1 were chosen so that $\mathrm{E}[\Phi]$ is a percentage of $\lim _{T \rightarrow \infty} \mathrm{E}[\Phi]$. The percentages were $90 \%$ (dashed-dotted), 95\% (dashed lines), 
TABLE I

UWB Channel Model PARAMETERS From [5, Table 2]

\begin{tabular}{cccrr} 
Channel & $C\left(\mathrm{~ns}^{-1}\right)$ & $R\left(\mathrm{~ns}^{-1}\right)$ & $\tau_{0}(\mathrm{~ns})$ & $s_{0}(\mathrm{~ns})$ \\
\hline $\mathrm{CM} 1$ & 0.0233 & 2.5 & 7.1 & 4.3 \\
$\mathrm{CM} 2$ & 0.4000 & 0.5 & 5.5 & 6.7 \\
$\mathrm{CM} 3$ & 0.0667 & 2.1 & 14.0 & 7.9 \\
$\mathrm{CM} 4$ & 0.0667 & 2.1 & 24.0 & 12.0 \\
\hline \multicolumn{7}{l}{ All models use $\sigma^{2}=\sigma_{1}^{2}+\sigma_{2}^{2}, \sigma_{1}=\sigma_{2}=3.3941}$.
\end{tabular}

Our numerical examples use $\Omega_{0}=-16 \mathrm{~dB}$.

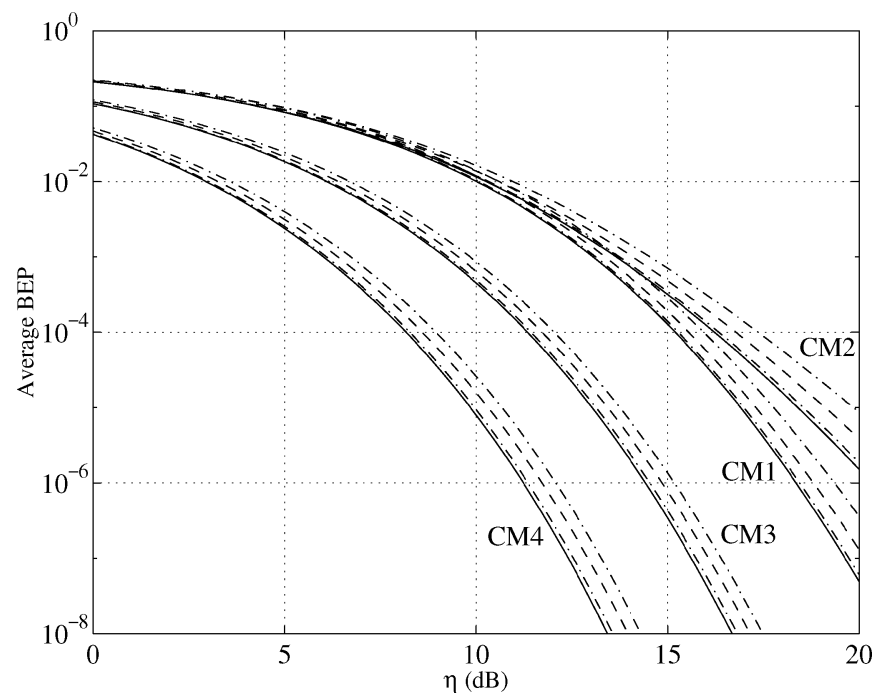

Fig. 1. Average BEP $P_{T}(\eta)$ as a function of $\eta$ for increasing values of $T$. The solid line is computed for $T=\infty$. The other values are listed in the text.

99\% (dashed-dotted), and 100\% (solid lines). We have from [6] that

$$
\begin{aligned}
& \mathrm{E}[\Phi]=\Omega_{0}\left(1+R s_{0}\left[1-e^{-T / s_{0}}\right]+C \tau_{0}\left[1-e^{-T / \tau_{0}}\right]\right. \\
&+C R\left\{\tau_{0} s_{0}\left[1-e^{-T / \tau_{0}}\right]-\frac{s_{0}^{2} \tau_{0}}{s_{0}-\tau_{0}}\right. \\
&\left.\left.\times\left[1-e^{-T\left(s_{0}-\tau_{0}\right) /\left(\tau_{0} s_{0}\right)}\right] e^{-T / s_{0}}\right\}\right) .
\end{aligned}
$$

It follows that

$$
\lim _{T \rightarrow \infty} \mathrm{E}[\Phi]=\Omega_{0}\left[1+R s_{0}+C \tau_{0}+C R s_{0} \tau_{0}\right] .
$$

Using the MATLAB function $f$ zero, it is easy to find the value of $T$ so that $\mathrm{E}[\Phi]$ is equal to a given fraction of $\lim _{T \rightarrow \infty} \mathrm{E}[\Phi]$.

As a check on our study, we compared the curves in Fig. 1 with the corresponding curves estimated by averaging $Q\left(\sqrt{\mathcal{E} \Phi /\left(4 \sigma_{n}^{2}\right)}\right)$ over 30000 realizations of the channel model (a time-consuming procedure). In all cases, the simulation results were quite close to the results using our method. A sample comparison for the 95\% curves is shown in Fig. 2.

Although [5] suggests using only 100 realizations, we have found considerable variation in simulated $P_{T}(\eta)$ curves even when using 3000 realizations. For each channel in Table I, we generated ten 3000-realization estimates of the dashed-line curve in Fig. 1. In each case, the two most extreme of the ten

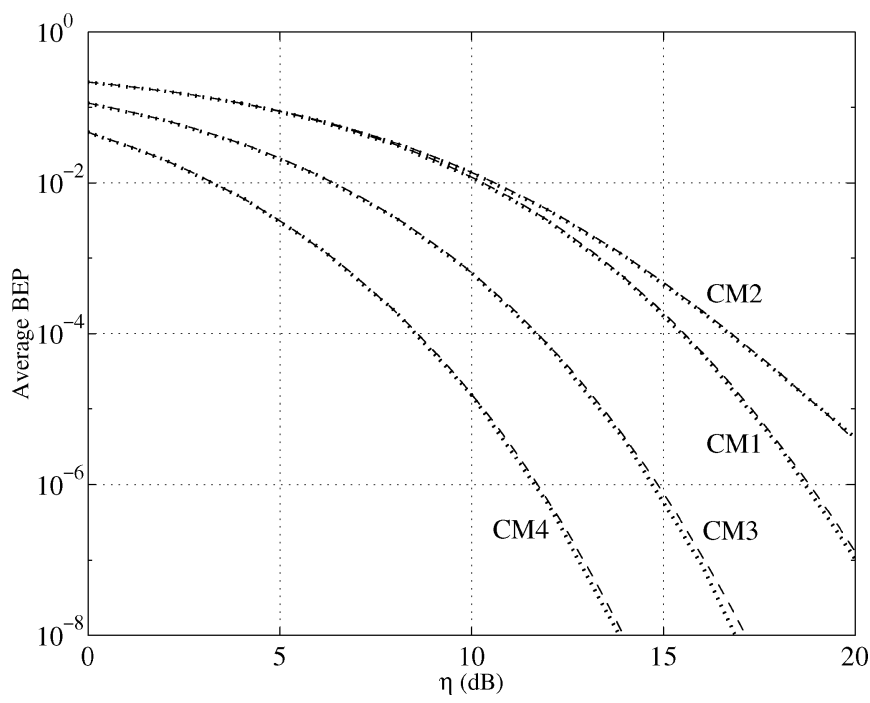

Fig. 2. Dotted lines result from using 30000 channel realizations to estimate $P_{T}(\eta)$ for the $95 \%$ values of $T$. The corresponding dashed lines are copied from Fig. 1 .

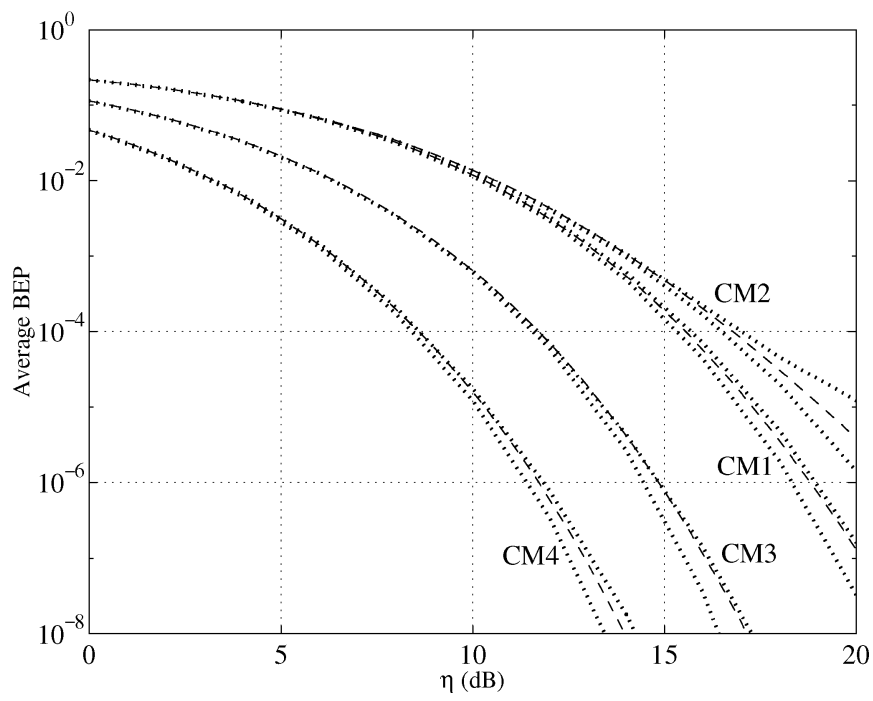

Fig. 3. Dotted lines result from using 3000 channel realizations to estimate $P_{T}(\eta)$ for the $95 \%$ values of $T$. The corresponding dashed lines are copied from Fig. 1.

estimates are denoted by the dotted lines in Fig. 3. The dashed line is copied from Fig. 1.

\section{CONCLUSION}

We have derived a computable formula for the average BEP of the IEEE 802.15.3a UWB channel model for finite observation windows. This formula can be quickly evaluated using Gaussian quadratures and can be used to quantify the tradeoff between the average BEP and the receiver observation window size. Our examples suggest that a receiver using an observation window that includes $99 \%$ of the expected energy in the channel gains has essentially the same performance as a receiver using an infinite observation window. Furthermore, the window size required for a given percentage is computable using nothing more complicated than the MATLAB command fzero. 


\section{APPENDIX \\ DERIVATION OF (14)}

We show that the three factors on the right-hand side in (13) are equal to the corresponding factors in (14).

\section{A. First Factor}

As noted at the beginning of Section III, to compute the average BEP, we take $\varphi(s, g)=g^{2} I_{[0, T]}(s)$. Hence, $\varphi\left(0, G_{0}\right)=$ $G_{0}^{2}$, where $G_{0}$ has the density $f_{0,0}(\cdot)$ defined in Section III. In particular, the first factor on the right-hand side in (13) reduces to $\mathrm{E}\left[e^{\theta G_{0}^{2}}\right]=\mathrm{E}_{0,0}\left[e^{\theta G^{2}}\right]$, which is given by (12), where an easy change of variable results in $M_{\sigma}\left(K_{0} \theta\right)$.

\section{B. Second Factor}

The second factor on the right-hand side in (13) involves $\Phi_{r 0}$. As shown in [6], $\Phi_{r 0}$ is a shot-noise random variable driven by a two-dimensional Poisson process with intensity function $\lambda_{r}\left(s, g \mid 0, G_{0}\right)$ given by (9). Hence, from [10, Ch. 3],

$$
\mathrm{E}\left[e^{\theta \Phi_{r 0}}\right]=\exp \left[\int_{0}^{\infty} \int_{-\infty}^{\infty}\left[e^{\theta \varphi(s, g)}-1\right] \lambda_{r}\left(s, g \mid 0, G_{0}\right) d g d s\right] .
$$

Since $\varphi(s, g)=g^{2} I_{[0, T]}(s)$, it is easy to compute

$$
\mathrm{E}\left[e^{\theta \Phi_{r 0}}\right]=e^{-R \psi_{0}\left(K_{0} \theta\right)}
$$

where for $\tau<T$,

$$
\psi_{\tau}(\theta):=\int_{\tau}^{T} 1-M_{\sigma}\left(\theta e^{-(s-\tau) / s_{0}}\right) d s .
$$

When $T<\infty$, make the change of variable $x=(s-\tau) /(T-\tau)$ to obtain (17). (This is in contrast to the change of variable $x=$ $\exp \left[-(s-\tau) / s_{0}\right]$ used to obtain (19) when $T=\infty$.)

\section{Third Factor}

The third factor on the right-hand side in (13) involves $\Phi_{\otimes}$. It is shown in the Appendix, Section D that

$$
\mathrm{E}\left[e^{\theta \Phi_{\otimes}}\right]=\exp \left[\int_{0}^{\infty} \int_{-\infty}^{\infty}\left[e^{k_{\theta}(\tau, \gamma)}-1\right] \lambda_{1}(\tau, \gamma) d \gamma d \tau\right]
$$

where

$$
k_{\theta}(\tau, \gamma):=\theta \gamma^{2} I_{[0, T]}(\tau)-R \psi_{\tau}\left(\theta K_{0} e^{-\tau / \tau_{0}}\right) .
$$

Using the formula for $k_{\theta}(\tau, \gamma)$ and (8), it is easy to show that where

$$
J(\theta):=\int_{0}^{T} 1-M_{\sigma}\left(\theta e^{-\tau / \tau_{0}}\right) \exp \left[-R \psi_{\tau}\left(\theta e^{-\tau / \tau_{0}}\right)\right] d \tau .
$$

When $T<\infty$, make the change of variable $x=\tau / T$ to obtain (18). (This is in contrast to the change of variable $x=e^{-\tau / \tau_{0}}$ used to obtain (20) when $T=\infty$.)

\section{Moment Generating Function of $\Phi_{\otimes}$}

As noted in [6, Appendix C], $\Phi_{\otimes}$ can be written in the form $\Phi_{\otimes}=V+P$, where, analogous to (7),

$$
V:=\int_{0}^{\infty} \int_{-\infty}^{\infty} \varphi(\tau, \gamma) N_{1}(d \tau \times d \gamma)
$$

and

$$
P:=\int_{0}^{\infty} \int_{-\infty}^{\infty} \varphi(s, g) N_{\times}(d s \times d g)
$$

where $N_{1}$ is the Poisson process with intensity function $\lambda_{1}(\tau, \gamma)$ in (8), and conditioned on the realization of $N_{1}, N_{\times}$is a Poisson process with intensity (cf. [6, App. B])

$$
m_{\times}(s, g)=\int_{0}^{\infty} \int_{-\infty}^{\infty} \lambda_{r}(s, g \mid \tau, \gamma) N_{1}(d \tau \times d \gamma) .
$$

In other words, $P$ has the conditional moment generating function

$$
\begin{aligned}
\mathrm{E} & {\left[e^{\theta P} \mid N_{1}(\cdot)\right] } \\
& =\exp \left[\int_{0}^{\infty} \int_{-\infty}^{\infty}\left(e^{\theta \varphi(s, g)}-1\right) m_{\times}(s, g) d s d g\right] \\
& =\exp \left[-R \int_{0}^{\infty} \int_{-\infty}^{\infty} \psi_{\tau}\left(\theta K_{0} e^{-\tau / \tau_{0}}\right) N_{1}(d \tau \times d \gamma)\right]
\end{aligned}
$$

where the last step uses the fact that $\varphi(s, g)=g^{2} I_{[0, T]}(s)$. Let us denote this last double integral by $\Psi$. Observe that $\Psi$ looks just like $V$ above, except with a different integrand. Using properties of conditional expectation,

$$
\mathrm{E}\left[e^{\theta(V+P)} \mid N_{1}(\cdot)\right]=e^{\theta V} \mathrm{E}\left[e^{\theta P} \mid N_{1}(\cdot)\right]=e^{\theta V-R \Psi}
$$

and then

$$
\mathrm{E}\left[e^{\theta(V+P)}\right]=\mathrm{E}\left[\mathrm{E}\left[e^{\theta(V+P)} \mid N_{1}(\cdot)\right]\right]=\mathrm{E}\left[e^{\theta V-R \Psi}\right] .
$$

$$
\mathrm{E}\left[e^{\theta \Phi_{\otimes}}\right]=e^{-C J\left(K_{0} \theta\right)}
$$


Now, both $V$ and $\Psi$ are shot-noise random variables driven by the same Poisson process $N_{1}$. By combining the integrands of the counting integrals, we obtain

$$
\mathrm{E}\left[e^{\theta V-R \Psi}\right]=\mathrm{E}\left[\exp \left\{\int_{0}^{\infty} \int_{-\infty}^{\infty} k_{\theta}(\tau, \gamma) N_{1}(d \tau \times d \gamma)\right\}\right]
$$

where $k_{\theta}(\tau, \gamma)$ was defined in (24). Since $N_{1}$ is a Poisson process with intensity function $\lambda_{1}(\tau, \gamma)$, this last expectation is given by (23) [10, Ch. 3].

\section{REFERENCES}

[1] M. Abramowitz and I. A. Stegun, Eds., Handbook of Mathematical Functions, With Formulas, Graphs, and Mathematical Tables. New York: Dover, 1970.

[2] A. Batra, J. Balakrishnan, G. R. Aiello, J. R. Foerster, and A. Dabak, "Design of a multiband OFDM system for realistic UWB channel environments," IEEE Trans. Microw. Theory Tech., vol. 52, no. 9, pp. 2123-2138, Sep. 2004.

[3] J. W. Craig, "A new, simple and exact result for calculating the probability of error for two-dimensional signal constellations," in Proc. IEEE Military Commun. Conf., McLean, VA, Oct. 1991, pp. 571-575.

[4] D. J. Daley and D. Vere-Jones, An Introduction to the Theory of Point Processes. New York: Springer, 1988.

[5] J. Foerster, Channel Modeling Sub-Committee report final IEEE Doc. P802.15-02/490r1-SG3a, 2003.

[6] J. A. Gubner and K. Hao, IEEE 802.15.3a UWB channel model as a two-dimensional augmented cluster process, submitted for publication.

[7] _ _ "An exact computable formula for the average bit-error probability of the IEEE 802.15.3a UWB channel model," in IEEE Int. UltraWideband Conf., 2005, pp. 142-146.

[8] M. Hamalainen, R. Tesi, and J. Iinatti, "UWB co-existence with IEEE 802.11a and UMTS in modified Saleh-Valenzuela channel," in Proc. Int. Ultrawideband Systems and Technologies Workshop, May 2004, pp. 45-59.

[9] S. Imada and T. Ohtsuki, "Pre-rake diversity combining form UWB systems in IEEE 802.15 UWB multipath model," in Proc. Int. Ultrawideband Syst. Technol. Workshop, May 2004, pp. 236-240.

[10] J. F. C. Kingman, Poisson Processes. Oxford, U.K.: Clarendon, 1993

[11] D. R. McKinstry and R. M. Buehrer, "UWB small scale channel modeling and system performance," in IEEE Veh. Technol. Conf., Oct. 2003, vol. 1, pp. 6-10.

[12] A. F. Molisch, J. R. Foerster, and M. Pendergrass, "Channel models for ultra-wideband personal area networks," IEEE Wireless Commun., vol. 10, no. 6, pp. 14-21, Dec. 2003.

[13] J. G. Proakis, Digital Communications, 4th ed. Boston, MA: McGraw-Hill, 2001.
[14] M. A. Rahman, S. Sasaki, J. Zhou, S. Muramatsu, and H. Kikuchi, "Performance evaluation of RAKE reception of ultra wideband signals over multipath channels from energy capture perspective," in Proc. Int. Ultrawideband Systems and Technologies Workshop, May 2004, pp. 231-235.

[15] A. Rajeswaran, V. S. Somayazulu, and J. R. Foerster, "Rake performance for a pulse based UWB system in a realistic UWB indoor channel," in Proc. IEEE Int. Commun. Conf., May 2003, vol. 4, pp. 2879-2883.

[16] A. A. M. Saleh and R. Valenzuela, "A statistical model for indoor multipath propagation," IEEE J. Select. Areas Commun., vol. SAC-5, no. 2, pp. 128-137, Feb. 1987.

[17] M. K. Simon and M. Alouini, "A unified approach to the performance analysis of digital communication over generalized fading channels," Proc. IEEE, vol. 86, no. 9, pp. 1860-1877, Sep. 1998.

[18] S. S. Tan, B. Kannan, and A. Nallanathan, "Ultra-wideband impulse radio systems with temporal and spatial diversities," in IEEE Veh. Technol. Conf., Oct. 2003, vol. 1, pp. 607-611.

[19] L. Zhiwei, B. Premkumar, and A. S. Madhukumar, "MMSE detection for high data rate UWB MIMO systems," in IEEE Veh. Technol. Conf., Sep. 2004, vol. 2, pp. 1463-1467.

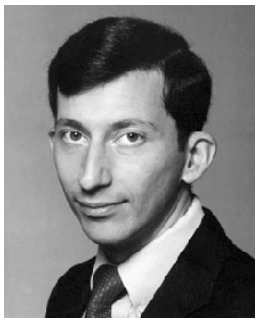

John A. Gubner (M'88) received the B.S., M.S., and $\mathrm{Ph} . D$. degrees in electrical engineering from the University of Maryland at College Park, in 1983, 1985, and 1988 , respectively.

From 1986 to 1988, he was a Graduate Fellow with the Systems Research Center (now the Institute for Systems Research), University of Maryland at College Park. Since 1988, he has been on the faculty of the Department of Electrical and Computer Engineering, University of Wisconsin-Madison. He authored the graduate textbook Probability and Random Processes for Electrical and Computer Engineers (Cambridge Univ. Press, 2006). His research interests include point processes and shot noise, information theory, wireless communications, and communication networks.

Dr. Gubner was the recipient of a 1985 IEEE Frank A. Cowan Scholarship for graduate study in communications.

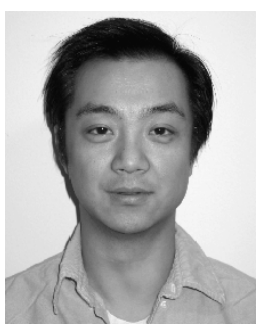

Kei Hao received the B.S. degree in electrical engineering from La Universidad de la Republica del Oriental del Uruguay, Montevideo, Uruguay, in 1998, the M.S. degree in electrical engineering from the University of Wisconsin-Milwaukee, in 2001, and is currently working toward the Ph.D. degree at the University of Wisconsin-Madison.

From 1998 to 2000, he was an Electrical Engineer with the South America Division, Schlumberger. While with the University of Wisconsin-Milwaukee, he was a Research Assistant with the Wireless and Signal Processing Laboratory and Center for Intelligent Maintenance Systems. His research interests include UWB communications, point-process theory, wireless communications, multiuser detection, information theory, and signal processing for communications. 